CARTA AL EDITOR

\section{EXPERIENCIA DEL USO DE TOCILIZUMAB EN PACIENTES CON COVID-19 ATENDIDOS EN UN HOSPITAL DE REFERENCIA DEL PERÚ}

\section{EXPERIENCE OF USING TOCILIZUMAB IN PATIENTS WITH COVID-19 FROM A REFERRAL HOSPITAL IN PERU}

\author{
Antonio Morales-Avalos (iD) 1,a Kenneth G. Vargas-Ponce ${ }^{11, b}$ \\ Jose J. Idrogo-Alfaro (iD) 1,b ,Juan A. Salas-López (1D) 1,2,a, \\ Félix K. Llanos-Tejada (1) 1,3,a
}

1 Servicio de Neumología, Hospital Nacional Dos de Mayo, Lima, Perú.

2 Facultad de Medicina Humana, Universidad Nacional Mayor de San Marcos, Lima, Perú.

3 Instituto de Investigación de Ciencias Biomédicas, Universidad Ricardo Palma Lima, Perú.

a $\quad$ Médico neumólogo; ${ }^{\mathrm{b}}$ médico residente de Neumología.

Sr. Editor: El SARS-CoV-2 es una nueva cepa de coronavirus que causa la COVID-19; los casos confirmados superaron los 100 millones de personas en todo el mundo, con más de 500 mil de muertes. Hasta el 10 de febrero del 2021, el Perú registró más de un millón de casos y 40 mil fallecidos ${ }^{(1)}$.

La neumonía por SARS-CoV-2 induce la liberación de citoquinas proinflamatorias (TNF- $\alpha$, IL-2, IL-6, factor estimulador de colonias de granulocitos y varias quimiocinas), las cuales provocan un estado hiperinflamatorio sistémico, llamado «tormenta de citoquinas» (2). Se ha reportado que los niveles plasmáticos de IL-6 en los pacientes con la COVID-19 son más altos de lo que generalmente se observa en una sepsis bacteriana y que están relacionadas con lesión pulmonar más grave ${ }^{(2)}$.

El tocilizumab es un anticuerpo monoclonal humanizado recombinante dirigido contra el receptor de interleucina-6 (IL-6), está aprobado por la Administración de Alimentos y Medicamentos (FDA, por sus siglas en inglés) de los Estados Unidos para el síndrome de liberación de citoquinas ${ }^{(3)}$, varios estudios de cohorte retrospectivos han mostrado mejoria en la neumonía y en los síntomas asociados en pacientes con COVID-19; sugiriendo que el tocilizumab en etapas tempra-

\footnotetext{
Citar como: Morales-Avalos A, Vargas-Ponce KG, Idrogo-Alfaro JJ, SalasLópez JA, Llanos-Tejada FK. Experiencia del uso de tocilizumab en pacientes con COVID-19 atendidos en un hospital de referencia del Perú. Rev Peru Med Exp Salud Publica. 2021;38(2):360-2. doi: https://doi.org/10.17843/ rpmesp.2021.382.6756.
}

Correspondencia: Kenneth Grenis Vargas Ponce; avenida Jesús María 973, Lima, Perú; grenisvp@gmail.com

Recibido: 16/11/2020 Aprobado: 24/02/2021 En línea: 26/03/2021 nas de la enfermedad puede asociarse con una respuesta favorable en pacientes con graves infecciones ${ }^{(2,4,5)}$.

El objetivo de este reporte es describir las características demográficas, clínicas, laboratoriales, radiológicas y el desenlace de pacientes con diagnóstico de COVID-19 que recibieron tocilizumab en el servicio de neumología del Hospital Nacional Dos de Mayo en Lima, Perú.

Se revisaron las historias clínicas de los pacientes hospitalizados desde el 1 de marzo hasta el 30 de julio del 2020, se incluyeron a todos los pacientes con diagnóstico de COVID-19 por RT-PCR en hisopado nasofaríngeo y/o dosaje de IgM/IgG sérico, y que cumplían los criterios para uso de tocilizumab según el protocolo del hospital (IL-6 mayor e igual a $40 \mathrm{pg} / \mathrm{ml} \mathrm{y} \mathrm{PaO}_{2} / \mathrm{FiO}_{2}$ menor a $200 \mathrm{mmHg}$ ), todos los pacientes recibieron una sola dosis de tocilizumab $(8 \mathrm{mg} / \mathrm{kg})$.

Los datos se analizaron de forma descriptiva mediante medidas de tendencia central, distribución de frecuencias, se usó la prueba exacta de Fisher para variables cualitativas y la U de Mann Whitney para variables cuantitativas.

Se evaluaron 31 pacientes de los cuales el $87,1 \%$ fueron de sexo masculino, la mediana de edad fue de 64 años, las comorbilidades más frecuentemente fueron: obesidad $(51,6 \%)$, diabetes $(25,8 \%)$ e hipertensión arterial $(19,4 \%)$. El 6,5\% no reportó ninguna comorbilidad.

La mediana del porcentaje de afectación radiológica fue de $61 \%$, la infección bacteriana sobreagregada y el ingreso a la unidad de cuidados intensivos se observó en el 16,1\% y $32,3 \%$, respectivamente, el $60 \%$ de pacientes ingresados fueron dados de alta, el $40 \%$ de pacientes fallecieron. Se encontró que el $9,1 \%$ de pacientes que recibieron tocilizumab con un tiempo de enfermedad menor a 11 días tuvo desenlace fatal, el 78,3\% de pacientes con frecuencia respiratoria menor a 36 respiraciones por minuto ( $\mathrm{rpm}$ ) previo al uso de tocilizumab fue dado de alta y el $63,6 \%$ de pacientes con frecuencia respiratoria mayor e igual a $30 \mathrm{rpm}$ posterior al uso de tocilizumab fallecieron.

Con relación a los resultados de laboratorio previo a la administración de tocilizumab, la medianas fueron: IL-6 $165 \mathrm{pg} / \mathrm{ml}$, linfocitos $733 \mathrm{cel} / \mathrm{mm}^{3}$, LDH $443 \mathrm{UI} / \mathrm{L}$, PCR 176 mg/L, Dímero D 0,7 mg/mL, TGO 68,5 U/L, TGP 70,5 U/L y $\mathrm{PaO}_{2} / \mathrm{FiO}_{2} 86 \mathrm{mmHg}$.

En el control de laboratorio, al quinto día posterior a la administración de tocilizumab, se encontró que el 25,8\% de pacientes presentaron un incremento de linfocitos, el $\mathrm{PaO}_{2} / \mathrm{FiO}_{2}$ fue menor en el grupo de fallecidos en comparación con los sobrevivientes $(68,5 \mathrm{mmHg}$ vs. $116 \mathrm{mmHg})$, el 21,4\% de los pacientes con una mediana de PCR de $157 \mathrm{mg} / \mathrm{dL}$ fallecieron en contraste con el $78,6 \%$ de pacientes con una mediana de PCR de $19 \mathrm{mg} / \mathrm{dL}$. La mediana de dímero D fue $1,5 \mathrm{mg} / \mathrm{mL}$, este valor fue mayor en los pacientes que fallecieron en relación con los sobrevivientes. La mediana de LDH fue de 409 $\mathrm{UI} / \mathrm{L}$, siendo menor en pacientes sobrevivientes en relación con fallecidos (296 UI/L vs. 706,5 UI/L) (Tabla 1). 
Tabla 1. Características demográficas, clínicas, radiológicas y laboratoriales con relación al desenlace de pacientes con la COVID-19 atendidos en un hospital de referencia del Perú.

\begin{tabular}{|c|c|c|c|c|}
\hline Características & $\begin{array}{c}\text { Total } \\
(\mathbf{n}=\mathbf{3 1})\end{array}$ & $\begin{array}{l}\text { Sobrevivientes } \\
\quad(n=21)\end{array}$ & $\begin{array}{l}\text { Fallecidos } \\
(\mathbf{n}=\mathbf{1 0})\end{array}$ & Valor de $p$ \\
\hline Edad, mediana (RIC) & $64(52-69)$ & $61(52-69)$ & $66(58-69)$ & $0,278^{a}$ \\
\hline Sexo masculino, n (\%) & $27(87,1)$ & $19(70,4)$ & $8(29,6)$ & $0,577^{\mathrm{b}}$ \\
\hline Sexo femenino, n (\%) & $4(12,9)$ & $2(50)$ & $2(50)$ & \\
\hline \multicolumn{5}{|l|}{ Comorbilidades, n (\%) } \\
\hline Obesidad & $16(51,6)$ & $11(68,7)$ & $5(31,3)$ & $0,528^{b}$ \\
\hline Diabetes & $8(25,8)$ & $5(62,5)$ & $3(37,5)$ & $0,483^{\mathrm{b}}$ \\
\hline Hipertensión & $6(19,4)$ & $4(66,7)$ & $2(33,3)$ & $0,568^{\mathrm{b}}$ \\
\hline Sin comorbilidad & $2(6,5)$ & $1(50,0)$ & $1(50,0)$ & $0,548^{\mathrm{b}}$ \\
\hline \multicolumn{5}{|l|}{ Infección bacteriana, n (\%) } \\
\hline Sí & $5(16,1)$ & $3(60,0)$ & $2(40,0)$ & $0,528^{\mathrm{b}}$ \\
\hline No & $26(83,9)$ & $18(69,2)$ & $8(30,8)$ & \\
\hline \multicolumn{5}{|l|}{ Ingreso a UCI, n (\%) } \\
\hline Sí & $32,3(10)$ & $6(60,0)$ & $4(40,0)$ & $0,405^{\mathrm{b}}$ \\
\hline No & $67,7(21)$ & $15(71,4)$ & $6(28,6)$ & \\
\hline Tiempo de enfermedad (días), mediana (RIC) & $12(8-15)$ & $10(7-14)$ & $13(13-15)$ & $0,082^{\mathrm{a}}$ \\
\hline \multicolumn{5}{|l|}{ Tiempo de enfermedad (días) previo a tocilizumab, n (\%) } \\
\hline$\leq 10$ días & $11(35,5)$ & $10(90,9)$ & $1(9,1)$ & $0,046^{\mathrm{b}}$ \\
\hline$>10$ días & $20(64,5)$ & $11(55,0)$ & $9(45,0)$ & \\
\hline Porcentaje de afectación radiológica, mediana (RIC) & $61(49-70,5)$ & $57(46-69)$ & $70(64-88)$ & $0,013^{\mathrm{a}}$ \\
\hline \multicolumn{5}{|l|}{ Frecuencia respiratoria } \\
\hline \multicolumn{5}{|l|}{ Antes de tocilizumab } \\
\hline$<36 \mathrm{rpm}$ & $23(74,2)$ & $18(78,3)$ & $5(21,7)$ & $0,048^{\mathrm{b}}$ \\
\hline$\geq 36 \mathrm{rpm}$ & $8(25,8)$ & $3(37,5)$ & $5(62,5)$ & \\
\hline \multicolumn{5}{|l|}{ Después de tocilizumab } \\
\hline$<30 \mathrm{rpm}$ & $20(64,5)$ & $17(85,0)$ & $3(15,0)$ & $0,009^{\mathrm{b}}$ \\
\hline$\geq 30 \mathrm{rpm}$ & $11(35,5)$ & $4(36,4)$ & $7(63,6)$ & \\
\hline \multicolumn{5}{|l|}{ IL- 6 (pg/ml), mediana (RIC) } \\
\hline Antes de tocilizumab & $165(49,3-368)$ & $135,5(49-347)$ & $190,5(100-449)$ & $0,645^{\mathrm{a}}$ \\
\hline \multicolumn{5}{|l|}{ Leucocitosis, células $/ \mathrm{mm}^{3}$, mediana (RIC) } \\
\hline Antes de tocilizumab & $11760(8499-15280)$ & $11110(8499-12340)$ & $14245(8525-15745)$ & $0,083^{\text {a }}$ \\
\hline Después de tocilizumab & $9280(6390-12825)$ & $8040(5740-10090)$ & $12825(10640-15250)$ & $0,008^{\mathrm{a}}$ \\
\hline \multicolumn{5}{|l|}{ Linfocitos, células $/ \mathrm{mm}^{3}$, mediana (RIC) } \\
\hline Antes de tocilizumab & $733(602-966)$ & $966(689-1074)$ & $886(796-1031)$ & $0,254^{\mathrm{a}}$ \\
\hline Después de tocilizumab & $771(548-1075)$ & $876(613-1042)$ & $448(390-811)$ & $0,283^{\mathrm{a}}$ \\
\hline \multicolumn{5}{|l|}{ Lactato deshidrogenasa (UI/L), mediana (RIC) } \\
\hline Antes de tocilizumab & $443(365-542)$ & $488,5(322-645)$ & $469,5(340-615)$ & $0,551^{\mathrm{a}}$ \\
\hline Después de tocilizumab & $409(332-572)$ & $296(189-409)$ & $706,5(560-910)$ & $0,026^{\mathrm{a}}$ \\
\hline \multicolumn{5}{|l|}{ Proteína $\mathrm{c}$ reactiva mg/L, mediana (RIC) } \\
\hline Antes de tocilizumab & $176(70-273)$ & $85(72-221,5)$ & $361(269-405)$ & $0,230^{\mathrm{a}}$ \\
\hline Después de tocilizumab & $25,5(5-43,5)$ & $19(5-32,5)$ & 157 (93-185) & $0,005^{\mathrm{a}}$ \\
\hline
\end{tabular}

AST: aspartato aminotransferasa, ALT: alanina aminotransferasa, rpm: respiraciones por minuto, RIC: rango intercuartílico

a Prueba U de Mann Whitney, ${ }^{\text {b }}$ prueba exacta de Fisher

(Continuá en la página 362) 
Tabla 1. Características demográficas, clínicas, radiológicas y laboratoriales con relación al desenlace de pacientes con la COVID-19 atendidos en un hospital de referencia del Perú. (Viene de la página 361)

\begin{tabular}{|c|c|c|c|c|}
\hline Características & $\begin{array}{c}\text { Total } \\
(\mathbf{n}=31)\end{array}$ & $\begin{array}{l}\text { Sobrevivientes } \\
(\mathrm{n}=\mathbf{2 1})\end{array}$ & $\begin{array}{c}\text { Fallecidos } \\
(\mathbf{n}=10)\end{array}$ & Valor de $p$ \\
\hline \multicolumn{5}{|l|}{ Procalcitonina, mediana (RIC) } \\
\hline Antes de tocilizumab & $0,11(0,08-0,30)$ & $0,1(0,07-0,10)$ & $0,18(0,11-0,30)$ & $0,230^{\mathrm{a}}$ \\
\hline Después de tocilizumab & $0,78(0,05-0,16)$ & $0,07(0,06-0,15)$ & $0,09(0,086-0,11)$ & $0,932^{\mathrm{a}}$ \\
\hline \multicolumn{5}{|c|}{$\mathrm{PaO}_{2} / \mathrm{FiO}_{2} \mathrm{mmHg}$, mediana (RIC) } \\
\hline Antes de tocilizumab & $86(65,5-135,5)$ & $97,0(79,5-135,5)$ & $73,5(60-91)$ & $0,036^{\mathrm{a}}$ \\
\hline Después de tocilizumab & $104(73,5-245,5)$ & $116(285,7-96,5)$ & $68,5(61-74)$ & $0,002^{\mathrm{a}}$ \\
\hline \multicolumn{5}{|l|}{ AST (U/L), mediana (RIC) } \\
\hline Antes de tocilizumab & $68,5(44,5-122,8)$ & $64(43-112)$ & $92(63-353)$ & $0,386^{\mathrm{a}}$ \\
\hline Después de tocilizumab & $74,5(46,3-103,8)$ & $55(40-105)$ & $92(74-93)$ & $0,640^{\mathrm{a}}$ \\
\hline \multicolumn{5}{|l|}{ ALT (U/L), mediana (RIC) } \\
\hline Antes de tocilizumab & $70,5(145,8-109,3)$ & $58(46-113)$ & $70(45-71)$ & $0,676^{\mathrm{a}}$ \\
\hline Después de tocilizumab & $89(156,3-137,8)$ & $92,5(53-139)$ & $72(60-124)$ & $0,581^{\text {a }}$ \\
\hline \multicolumn{5}{|c|}{ Ferritina (ug/mL), mediana (RIC) } \\
\hline Antes de tocilizumab & $1145(887-1591)$ & $479(278-680)$ & $1578(950-1793)$ & $0,447^{\mathrm{a}}$ \\
\hline Después de tocilizumab & $656(226-995)$ & $216(84-348)$ & $952,5(650-1000)$ & $0,321^{\text {a }}$ \\
\hline \multicolumn{5}{|c|}{ Dímero D (mg/mL), mediana (RIC) } \\
\hline Antes de tocilizumab & $0,7(0,4-1,8)$ & $1,2(0,64-1,88)$ & $0,6(0,55-2,35)$ & $0,681^{\mathrm{a}}$ \\
\hline Después de tocilizumab & $1,5(0,7-3,5)$ & $2,3(1,39-3,43)$ & $1,7(0,67-7,3)$ & $0,362^{\mathrm{a}}$ \\
\hline
\end{tabular}

AST: aspartato aminotransferasa, ALT: alanina aminotransferasa, rpm: respiraciones por minuto, RIC: rango intercuartilico

${ }^{\text {a }}$ Prueba U de Mann Whitney, ${ }^{\text {b }}$ prueba exacta de Fisher

Las limitaciones de este estudio incluyen su naturaleza retrospectiva, la falta de un grupo control y el limitado tamaño de muestra. Todos los pacientes recibieron el esquema de tratamiento establecido por el hospital (corticoides y enoxaparina), es posible que los beneficios observados en este estudio se debieran al uso de estos medicamentos, también es posible que los pacientes que fallecieron tenían una enfermedad más severa y los que sobrevivieron tenían menor severidad de la enfermedad. Al ser una experiencia de un solo centro hospitalario, esto podría limitar la aplicabilidad a otros entornos.

En conclusión, podemos mencionar que el uso de anticuerpos monoclonales (tocilizumab) en etapas tempranas de la enfermedad (10 días) podría ser beneficiosa; asimismo, mejoras en la frecuencia respiratoria, $\mathrm{PCR}, \mathrm{DHL}$ y $\mathrm{PaO}_{2} / \mathrm{FiO}_{2}$ podrían ser indicadores de buena respuesta.

Es necesario publicar esta carta para que exista un registro de la experiencia del uso de tocilizumab en Perú durante esta pandemia. Actualmente este medicamento no se considera una alternativa para los casos graves de la COVID-19, pues su uso no está aprobado por falta de evidencia de efectividad frente a esta enfermedad.

Criterios éticos: Los autores declaran que este estudio forma parte de los datos recolectados en el protocolo «Entendiendo la pandemia COVID-19 en el Perú: Evaluación de la Cohorte Prospectiva del Hospital Nacional Dos de Mayo», aprobado en el Hospital Nacional Dos de Mayo con documento de Evaluación N º 018-1-2020CEIBHNDM y con aprobación del Comité de Ética de la Facultad de Medicina de la Universidad Ricardo Palma con código PI-019-2020.

Contribuciones de autoría: AMA, KVP, JSL, FLLT, JIA han participado en la concepción del artículo y en la recolección de datos, redacción y aprobación de la versión final.

Financiamiento: Autofinanciado.

Conflictos de interés: Los autores declaran no tener conflictos de interés.

\section{REFERENCIAS BIBLIOGRÁFICAS}

1. COVID-19 Map [Internet]. Johns Hopkins Coronavirus Resource Center. [citado 10 de febrero de 2021]. Disponible en: https://coronavirus. jhu.edu/map.htm.

2. Levi M. Tocilizumab for severe COVID-19: A promising intervention affecting inflammation and coagulation. Eur J Intern Med. 2020;76:212. doi: $10.1016 /$ j.ejim.2020.05.018.
3. Le RQ, Li L, Yuan W, Shord SS, Nie L, Habtemariam BA, et al. FDA Approval Summary: Tocilizumab for Treatment of Chimeric Antigen Receptor T Cell-Induced Severe or Life-Threatening Cytokine Release Syndrome. Oncologist. 2018;23(8):943-7. doi: 10.1634/theoncologist.2018-0028.

4. Furlow B. COVACTA trial raises questions about tocilizumab's benefit in COVID-19. Lancet Rheumatol. 2020;2(10):e592. doi: 10.1016/S26659913(20)30313-1.

5. Lan S-H, Lai C-C, Huang H-T, Chang S-P, Lu L-C, Hsueh P-R. Tocilizumab for severe COVID-19: a systematic review and meta-analysis. Int J Antimicrob Agents. 2020;56(3):106103. doi: 10.1016/j.ijantimicag.2020.106103. 